\title{
ON THE ACCURACY OF SPECTRAL CLASSIFICATIONS OF MAIN-SEQUENCE STARS
}

\author{
W. GLIESE \\ Astronomisches Rechen-Institut, Heidelberg, F.R.G.
}

\begin{abstract}
By examining the observed dispersion in (colour, spectral type) relations, classification errors have been derived from the data of nearby stars. The comparisons of the colour deviations observed in spectral regions of large variations of colour with type with the deviations in regions of small variations give the following standard errors in units of a tenth of a spectral class: For $\mathrm{K}$ dwarfs \pm 0.6 (MK), \pm 1.2 (Mt. Wilson), \pm 0.7 (Kuiper); for early $\mathrm{M}$ dwarfs \pm 0.9 : (MK), \pm 0.7 (Mt. Wilson), \pm 0.5 : (Kuiper); and for late $\mathrm{M}$ dwarfs +0.7 (Kuiper).
\end{abstract}

In the preceding paper, Jaschek has mentioned an estimated standard error of MK classifications of \pm 0.6 subclasses which has been given in a publication on mean relations between spectral types and photoelectric colours of nearby main-sequence stars (Gliese, 1971). The preliminary values of the accuracy of spectral classifications published there have been superseded by the results of a more refined investigation shown in the following. C. Jaschek has also demonstrated the method and the difficulties encountered when using the variation of colour with spectral type and the observed dispersions for deriving accidental errors in the classifications.

In the above cited publication 12 mean relations between MK, Mt. Wilson, and Kuiper types and the colours $B-V, U-B,(U-B)_{\text {Cape }}$, and $R-I$ have been derived using the data of the 'Catalogue of Nearby Stars' (Gliese, 1969) which have been supplemented by further data collected in card catalogue form:

$$
\begin{array}{ccc}
B-V \text {, MK type } & B-V, \text { Mt.Wilson type } & B-V, \text { Kuiper type } \\
U-B \text {, MK type } & U-B, \text { Mt.Wilson type } & U-B, \text { Kuiper type } \\
(U-B)_{\mathrm{C}} \text {, MK type } & (U-B)_{\mathrm{C}} \text {, Mt.Wilson type } & (U-B)_{\mathrm{C}}, \text { Kuiper type } \\
R-I, \text { MK type } & R-I, \text { Mt.Wilson type } & R-I, \text { Kuiper type }
\end{array}
$$

As an example Figure 1 shows the smoothed curve of the ( $U-B$, Kuiper type) relation. It is obvious that classification errors produce an additional dispersion in the colours which increases with increasing slope of the curve.

The principle of this method consists in comparisons of observed colour deviations in spectral regions of large variations of colour with type with the deviations in regions of small variations. In the ( $U-B$, Kuiper type) relation deviations in the K-dwarf region should be compared with those in the $G$ or $F$ or $A$ classes.

But before investigating how this method works a few remarks should be made about the spectral data used. Figure 2 shows the frequency of types from F0 to the late $\mathrm{M}$ dwarfs among the stars nearer than $22 \mathrm{pc}$. For $\mathrm{K}$ and $\mathrm{M}$ stars Kuiper has given numerous intermediates as $\mathrm{K} 4+, \mathrm{K} 5+$, and so on. The original MK system (the MKK) had a framework of only some special types; but in the mean time nearly 


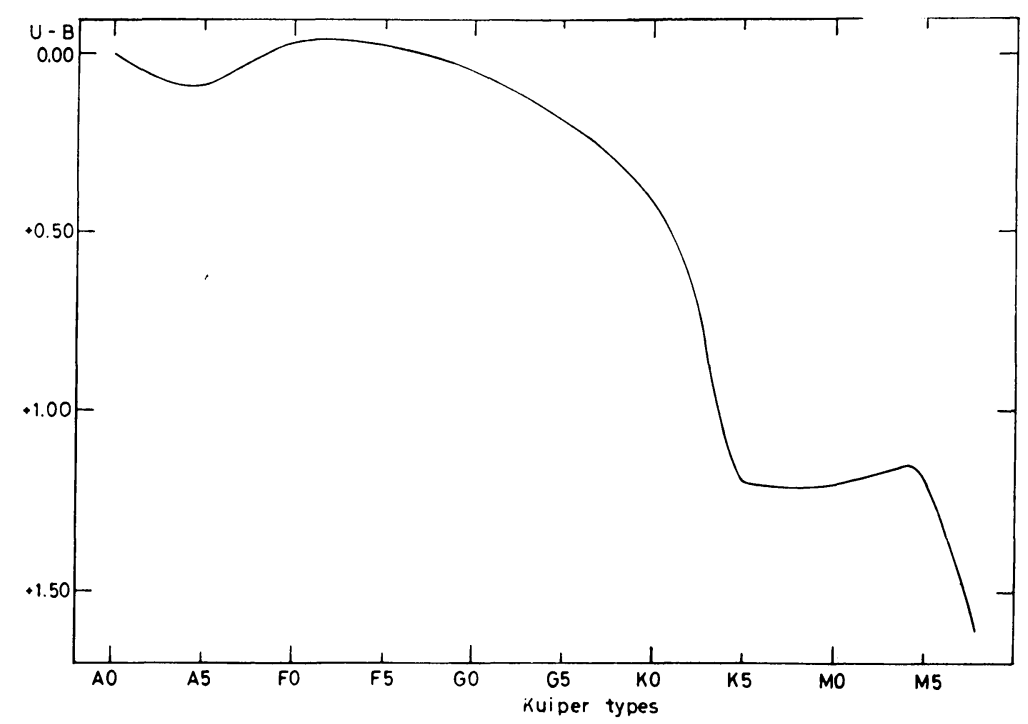

Fig. 1. ( $U-B$, Kuiper type) relation of nearby main-sequence stars.

every tenth of a class is found in the literature. But even now we see the preference for the original types.

The main source for MK types has been the 'Catalogue of Stellar Spectra Classified in the Morgan-Keenan System' by Jaschek et al. (1964). Objects with dubious luminosity classifications have been excluded. The material used here consists only of stars lying on the main sequence. If different classifications by various observers were available the one type seeming best fitted has been used. Therefore it may be expected that the standard errors derived here will be somewhat smaller than those found by Jaschek and Jaschek (1966).

Nearly all Mt. Wilson types have been taken from the General Catalogue of Stellar Radial Velocities (Wilson, 1953). Mostly these types agree with the classification of 1935 (Adams et al.) investigated by Butler and Thackeray (1940).

Kuiper's types are found in various publications, mainly in his list of stars nearer than 10 parsecs (1942) and in the General Catalogue of Trigonometric Stellar Parallaxes (Jenkins, 1952, 1963). Further red dwarf data were made available by private communication.

In the 12 (colour, spectral type) relations the observed standard deviations (s.d.) of the colours have been derived in various spectral regions; the s.d. result from observational errors in colour $\varepsilon_{c}$, from observational errors in spectral type $\varepsilon_{t}$, and from a 'cosmic dispersion' $\sigma$. As we are dealing with nearby stars only no further dispersion in colour by different effects of the interstellar matter will occur.

Many of the colour data used here are mean values of various measurements. Their errors are small and $\varepsilon_{c}$ is nearly insignificant, namely of the order \pm 0.01 or \pm 0.02 .

The classification error, $\varepsilon_{t}$, produces an additional contribution, $\varepsilon_{s}$, to the observed 


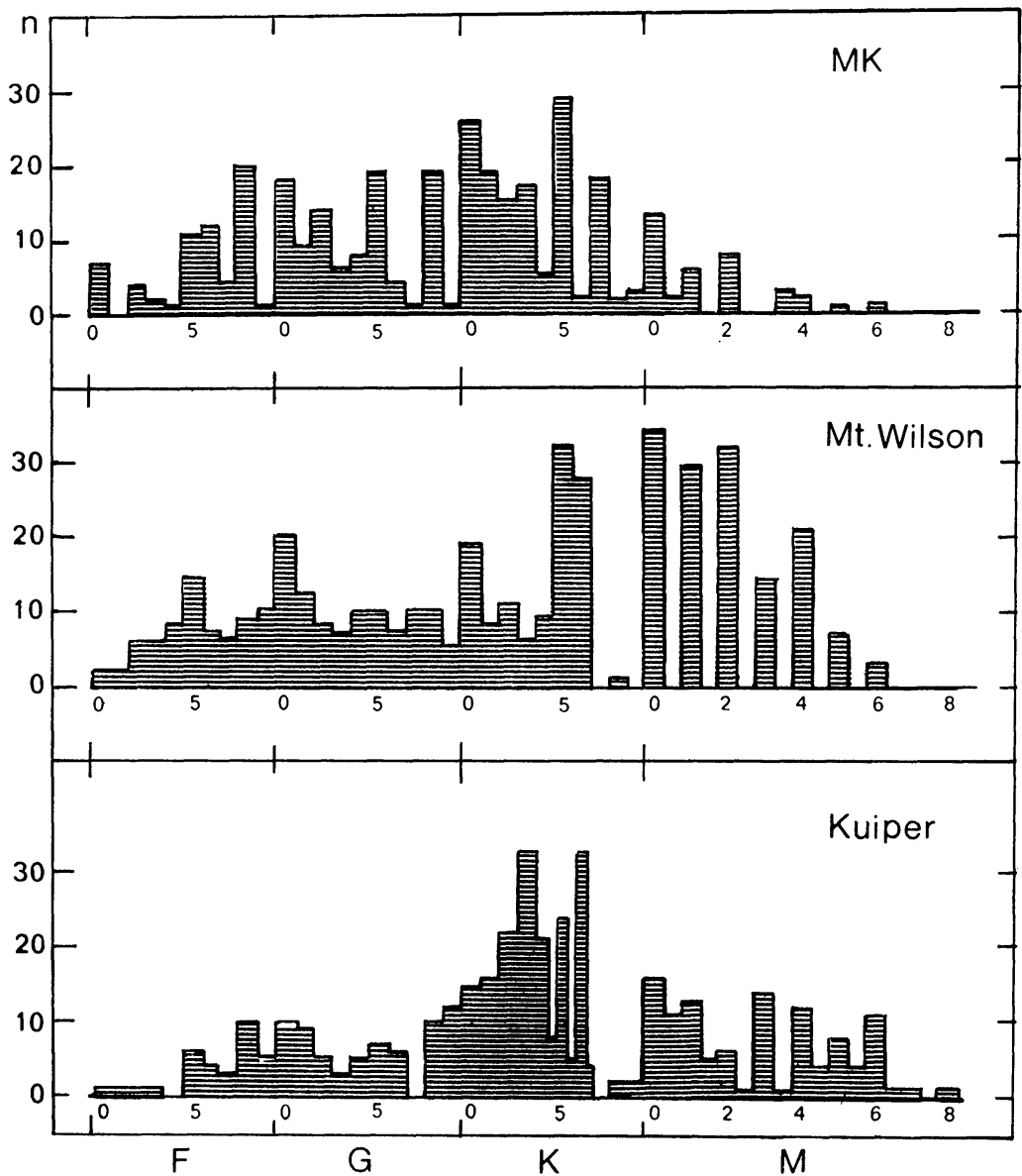

Fig. 2. Frequencies of the various spectral types of nearby stars.

standard deviations, which is $\varepsilon_{s}=\varepsilon_{t} v_{c}$, where $\varepsilon_{t}$ is given in units of a tenth of a spectral type and $v_{c}$ is the variation of colour with a tenth of a spectral class: $v_{c}=$ $\Delta$ colour / 0.1 spectral class.

Since there is no one to one correlation between spectral type and colour, we define $\sigma$ as the dispersion which occurs even after elimination of all observational errors; that is, the true colour dispersion in a (colour, spectral type) relation.

For each spectral region (s.d.) $)^{2}=\varepsilon_{c}^{2}+\varepsilon_{s}^{2}+\sigma^{2}$ has been derived from the observations. Substracting an estimated value of the square of the observational error, $\varepsilon_{c}$, a quantity $l$ is introduced by $l^{2}=(\text { s.d. })^{2}-\varepsilon_{c}^{2}$, or $l^{2}=\sigma^{2}+\varepsilon_{t}^{2} v_{c}^{2}$. $\sigma$ and $\varepsilon_{t}$ are unknown; probably both quantities vary with spectral type.

Figures 3-5 show in the 12 relations the observed $l^{2}$ (solid lines) together with the squares of the colour variation $v_{c}^{2}$ (dashed lines). Obviously in all diagrams there is a strong correlation.

From $\mathrm{A}$ to $\mathrm{G}$ the variation of colour with type is small. Normally, the solid lines 


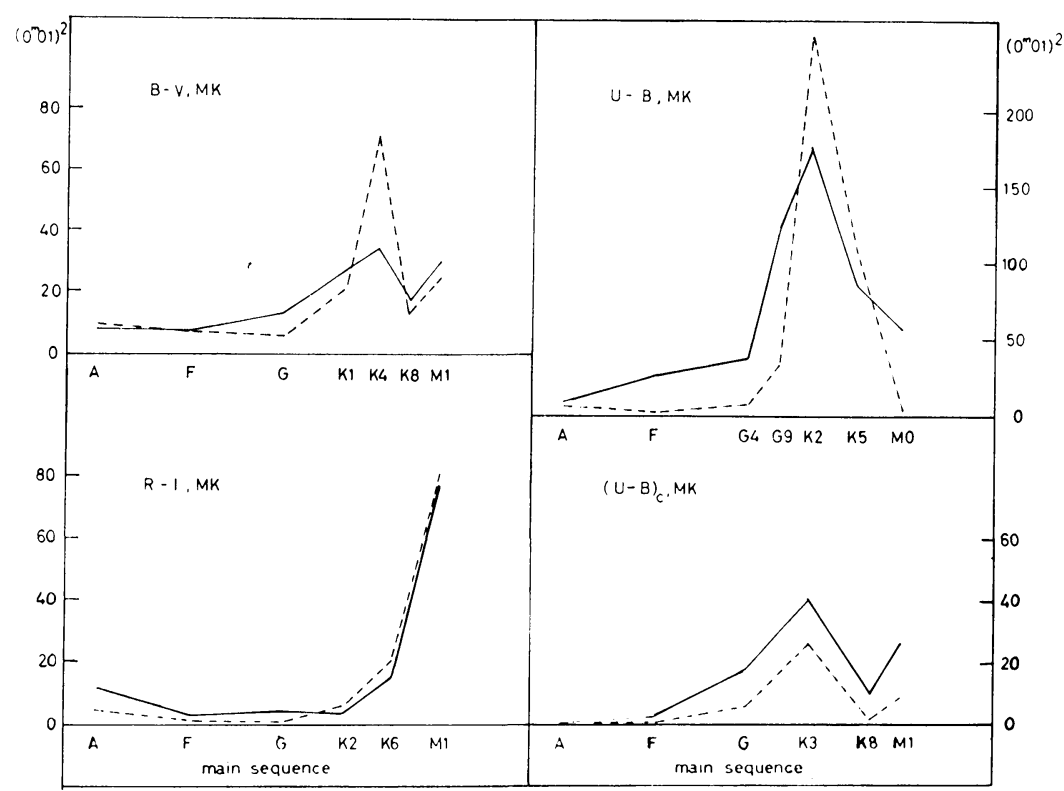

Fig. 3. Comparison between the colour dispersions observed in (colour, MK type) relations with the variation of colour with type for nearby main-sequence stars. Solid line: Squares of the dispersions observed in different spectral regions. Dashed line: Squares of the variation of colour for 0.1 spectral class. Unit for colours: 0.01 .

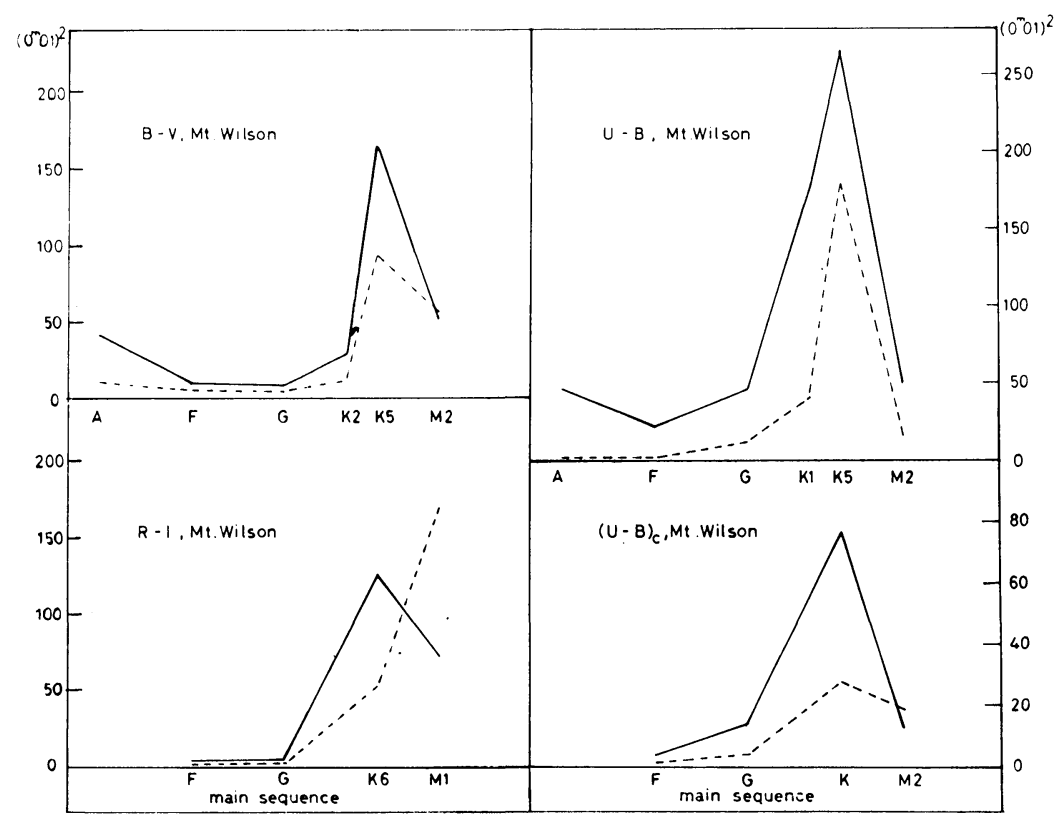

Fig. 4. Comparison between the colour dispersions observed in (colour, Mt. Wilson type) relations with the variation of colour with type for nearby main-sequence stars. Solid line: Squares of the dispersions observed in different spectral regions. Dashed line: Squares of the variation of colour for 0.1 spectral class. Unit for colours: 0.01 . 


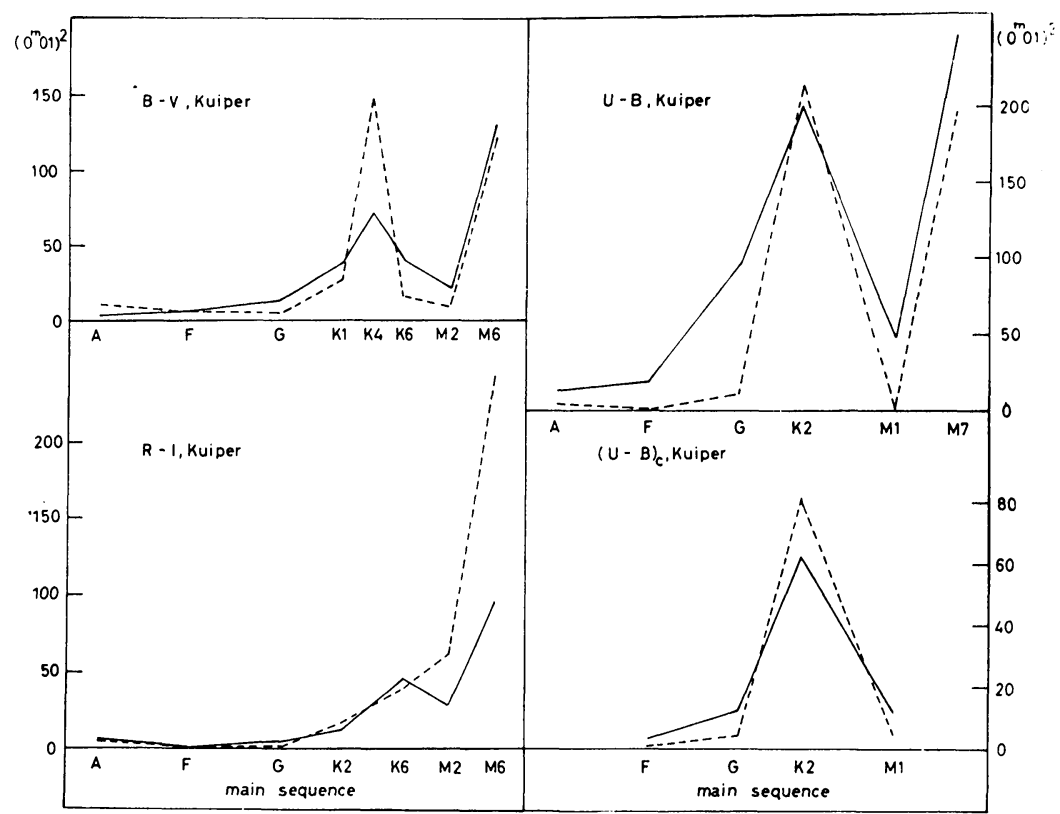

Fig. 5. Comparison between the colour dispersions observed in (colour, Kuiper type) relations with the variation of colour with type for nearby main-sequence stars. Solid line: Squares of the dispersions observed in different spectral regions. Dashed line: Squares of the variation of colour for 0.1 spectral class. Unit for colours: 0.01 .

are somewhat above the dashed values-a result which seems to be caused mainly by the $\sigma^{2}$. This region is not suitable for deriving the errors $\varepsilon_{t}$. Large coefficients $v_{c}^{2}$ are in the $\mathrm{K}$ and $\mathrm{M}$ star regions.

The limited number of objects does not allow an exact separation of the various quantities contributing to the observed dispersions. A preliminary view of these diagrams shows

(1) in the MK classification, $\varepsilon_{t}$ of the $\mathrm{K}$ types is smaller than 0.1 spectral class except in the $\left[(U-B)_{\mathrm{C}}, \mathrm{MK}\right.$ type $]$ diagram which has only low weight. For the early M dwarfs $\varepsilon_{t}$ is near a tenth of a spectral class.

(2) in the Mount Wilson classification, $\varepsilon_{t}$ is larger than one in class $\mathrm{K}$ but decreases for the early $M$ dwarfs.

(3) Kuiper's classification shows an $\varepsilon_{t}$ somewhat smaller than 0.1 spectral type.

To get precise values of the errors, differences between the quantities in regions of large variation $v_{c}$ of colour with type and $v_{c}$ in regions with low variations have been computed; for example

$$
\left(l^{2}\right)_{\mathrm{K}}-\left(l^{2}\right)_{\mathrm{G}}=\left(\sigma^{2}\right)_{\mathrm{K}}-\left(\sigma^{2}\right)_{\mathrm{G}}+\left(\varepsilon_{t}^{2}\right)_{\mathrm{K}}\left(v_{c}^{2}\right)_{\mathrm{K}}-\left(\varepsilon_{t}^{2}\right)_{\mathrm{G}}\left(v_{c}^{2}\right)_{\mathrm{G}}
$$

In these adjacent regions $\left(\sigma^{2}\right)_{\mathrm{K}}-\left(\sigma^{2}\right)_{\mathrm{G}}$ will be negligible and $\left(\varepsilon_{t}\right)_{\mathrm{G}}$ will be approximately equal to $\left(\varepsilon_{t}\right)_{\mathrm{K}}$ : 
$\left(\varepsilon_{t}\right)_{\mathrm{G}}=\left(\varepsilon_{t}\right)_{\mathrm{K}}+y \quad$ where $y$ is a small quantity: $|y| \ll 1$

$\left(\varepsilon_{t}^{2}\right)_{\mathrm{G}} \sim\left(\varepsilon_{t}^{2}\right)_{\mathrm{K}}+2\left(\varepsilon_{t}\right)_{\mathrm{K}} y$.

In the first approximation $\Delta \sigma^{2}=\left[\left(\sigma^{2}\right)_{\mathrm{K}}-\left(\sigma^{2}\right)_{\mathrm{G}}\right]$ and $y$ are supposed equal to zero:

$$
\left(\varepsilon_{t}^{2}\right)_{\mathrm{K}}=\frac{\left(l^{2}\right)_{\mathrm{K}}-\left(l^{2}\right)_{\mathrm{G}}}{\left(v_{c}^{2}\right)_{\mathrm{K}^{\prime}}-\left(v_{c}^{2}\right)_{\mathrm{G}}} .
$$

For example, the results by Butler and Thackeray (1940) show that the mean errors in the Mt. Wilson classification do not vary from $\mathrm{G}$ to $\mathrm{K}$ dwarfs. In this way approximate $\left(\varepsilon_{t}\right)_{\mathrm{K}}$ can be derived from a comparison with the dispersions in the $\mathrm{G}$ region and also in other spectral regions $(\mathrm{A}, \mathrm{F}, \ldots)$.

As an example Table I shows the approximate values of $\varepsilon_{t}$ in the classification of $\mathrm{K}$ dwarfs (mean type $\mathrm{K} 4$ or $\mathrm{K} 5$ ) as derived from the dispersions in the ( $B-V$, spectral type) relations. The last columns give relative weights in an arbitrary system. The weight of such a determination depends on the number of stars in the compared regions and on the value of the denominator which should not be too small. In the MK and in the Kuiper classifications the approximate values are slightly decreasing from A to $\mathrm{G}$; the same trend is observed in the $(U-B, \mathrm{MK}$ type) and ( $U-B, \mathrm{Kuiper}$ type) relations but not in the Mt. Wilson system. Such variations can be explained by an increase of the cosmic dispersion $\sigma$ from $A$ to $G$ whereas the true mean errors $\varepsilon_{t}$ probably do not vary appreciably in this spectral region from $A$ to $K(y \sim 0)$. In the $\left(R-I\right.$, spectral type) relations no trend in the approximate $\varepsilon_{t}$ is observed.

\section{TABLE I}

Approximate values of the classification errors $\varepsilon^{\prime}{ }_{t}$ of $\mathrm{K}$ dwarfs (mean type: $\mathrm{K} 4$ or $\mathrm{K} 5$; unit: 0.1 spectral type; $p$ : relative weight)

\begin{tabular}{|c|c|c|c|c|c|c|}
\hline \multirow{2}{*}{$\begin{array}{l}\text { Comparison } \\
\text { region }\end{array}$} & \multicolumn{2}{|l|}{ MK types } & \multicolumn{2}{|c|}{ Mt. Wilson types } & \multicolumn{2}{|c|}{ Kuiper types } \\
\hline & $\varepsilon_{t}^{\prime}$ & $p$ & $\varepsilon_{t}^{\prime}$ & $p$ & $\varepsilon_{t}^{\prime}$ & $p$ \\
\hline $\mathrm{A}$ & +0.66 & 0.5 & +1.21 & 0.6 & +0.71 & 1.0 \\
\hline $\mathrm{F}$ & 0.65 & 2.1 & 1.32 & 4.4 & 0.68 & 4.1 \\
\hline G & 0.57 & 3.5 & 1.32 & 6.1 & 0.64 & 8.3 \\
\hline $\mathbf{K} 1$ & 0.37 & 1.6 & 1.28 & 2.5 & 0.54 & 5.4 \\
\hline K7 & 0.55 & 0.7 & & & 0.50 & 4.8 \\
\hline M1 & 0.32 & 0.7 & 1.73 & 3.3 & 0.60 & 10.0 \\
\hline
\end{tabular}

Corresponding series of approximate values of $\varepsilon_{t}$ have been computed from the dispersions in all 12 relations and also for other spectral regions with the mean types $\mathrm{K} 1$, K7, M1 ... M6. From the trends of these $\varepsilon_{t}$ the true values of the mean errors are estimated making allowance for the different weights. The combination of all results gives the values in Table II.

But these results need some qualifying remarks; the complications in this method arise from the details. Spectral types are discrete points along a continuously filled 
TABLE II

Mean errors $\varepsilon_{t} t$ of spectral classifications, still affected by the 'slope effect' (Unit: 0.1 spectral type; $p$ : relative weights)

\begin{tabular}{|c|c|c|c|c|c|c|c|}
\hline \multirow[t]{2}{*}{ Spectral class } & \multirow[t]{2}{*}{ Colour } & \multicolumn{2}{|c|}{ MK types } & \multicolumn{2}{|c|}{ Mt. Wilson types } & \multicolumn{2}{|c|}{ Kuiper types } \\
\hline & & $\varepsilon_{t}^{\prime}$ & $p$ & $\overline{\varepsilon_{t}^{\prime}}$ & $p$ & $\overline{\varepsilon^{\prime} t}$ & $p$ \\
\hline \multirow[t]{4}{*}{$\mathbf{K}$} & $B-V$ & \pm 0.6 & 11 & \pm 1.4 & 17 & \pm 0.65 & 36 \\
\hline & $U-B$ & 0.7 & 22 & 1.2 & 35 & 0.85 & 56 \\
\hline & $(U-B)_{\mathrm{C}}$ & 1.1 & 1 & 1.6 & 0.4 & 0.8 & 2 \\
\hline & $R-I$ & 0.8 & 0.5 & 1.6 & 1 & 1.05 & 2 \\
\hline $\mathbf{K}$ & mean & \pm 0.7 & 35 & \pm 1.25 & 54 & \pm 0.8 & 96 \\
\hline \multirow[t]{4}{*}{ early $M$} & $B-V$ & \pm 1.0 & 1 & \pm 0.8 & 14 & & \\
\hline & $U-B$ & & & 1.2 & 1 & & \\
\hline & $(U-B)_{\mathrm{C}}$ & & & 0.7 & 0.1 & & \\
\hline & $R-I$ & 1.0 & 2.5 & 0.7 & 14 & \pm 0.6 & 2.5 \\
\hline early $\mathbf{M}$ & mean & \pm 1.0 & 4 & \pm 0.8 & 29 & \pm 0.6 & 2.5 \\
\hline \multirow[t]{3}{*}{ late $\mathbf{M}$} & $B-V$ & & & & & \pm 1.0 & 6 \\
\hline & $U-B$ & & & & & 1.0 & 4 \\
\hline & $R-I$ & & & & & 0.6 & 14 \\
\hline late $\mathbf{M}$ & mean & & & & & \pm 0.8 & 24 \\
\hline
\end{tabular}

sequence of spectra. Type $t_{i}$ is assigned to all spectra from $t_{i-1 / 2}$ to $t_{i+1 / 2}$ with the mean colours between $c_{i-1 / 2}=\frac{1}{2}\left(c_{i-1}+c_{i}\right)$ and $c_{i+1 / 2}=\frac{1}{2}\left(c_{i}+c_{i+1}\right)$. Therefore, even if all spectra are classed correctly (and $\sigma$ is equal to zero) a dispersion in colour will be observed. In a homogeneous filled sequence the average square of the colour deviations originating in this effect is one sixth of the square of the colour variation with a tenth of a spectral class: $\frac{1}{6} v_{c}^{2}$. This percentage is included in the $\varepsilon_{t}$ in Tables I and II. Its exact elimination seems to be difficult as this dispersion has no Gaussian distribution and only a limited number of objects is available. But it may be estimated that the values of the classification errors $\varepsilon_{t}$ in Table II have to be diminished by at most 0.1 .

The elimination of this 'slope effect' from the results in Table II gives the true values of the standard errors in classification which are listed in Table III.

TABLE III

Standard errors $\varepsilon_{t}$ of spectral classifications (Unit: 0.1 spectral type)

\begin{tabular}{lccc}
\hline Spectral class & MK & Mt. W. & Kuiper \\
\hline K & \pm 0.6 & \pm 1.2 & \pm 0.7 \\
early M & $0.9:$ & 0.7 & $0.5::$ \\
late M & & & 0.7
\end{tabular}


A comparison of the data in the three systems shows the superiority of the MK classification in the range of the $\mathrm{K}$ dwarfs. The value \pm 0.6 agrees fairly well with the accuracy derived by C. Jaschek and M. Jaschek (1966). $\varepsilon_{t}= \pm 1.2$ for the Mt. Wilson system confirms the result by Butler and Thackeray (1940). It is very interesting that the early $\mathbf{M}$ dwarfs have been classified with smaller errors than the Mt. Wilson $\mathrm{K}$ dwarfs even though their apparent magnitudes normally are fainter than those of the $\mathrm{dK}$ stars. The relatively high accuracy of the late $\mathrm{M}$ types classified by Kuiper should be emphasized.

An application of this method to other luminosity classes such as subgiants or giants will be complicated by the reddening effects.

Errors in the luminosity classification cannot be investigated. C. Jaschek and M. Jaschek (1966) have given estimates for the MK classes. The nearby-star data indicate that among $\mathrm{F}$ and $\mathrm{G}$ dwarfs and subgiants the percentage of dubious luminosity classifications in the Mt. Wilson system is nearly twice that of the MK system.

Concluding this report on the accuracy of classifications derived from nearby-star data, it should be pointed out that the errors given here are not valid for MK classifications on objective prism plates.

\section{Acknowledgements}

The author is indebted to Dr R. Laubscher for his assistance in the preparation of the English version of the text.

\section{References}

Adams, W. S., Joy, A. H., Humason, M. L., and Brayton, A. M.: 1935, Astrophys. J. 81, 187; Contr. Mt. Wilson Obs., No. 511.

Butler, H. E. and Thackeray, A. D.: 1940, Monthly Notices Roy. Astron. Soc. 100, 450.

Gliese, W.: 1969, Veröffentl. Astron. Rechen-Inst. Heidelberg, No. 22.

Gliese, W.: 1971, Veröffentl. Astron. Rechen-Inst. Heidelberg, No. 24.

Jaschek, C., Conde, H., and de Sierra, A. C.: 1964, Obs. Astron. Univ. Nac. La Plata Ser. Astron. 28 (2).

Jaschek, C. and Jaschek, M.: 1966, in K. Lodén, L. O. Lodén, and U. Sinnerstad (eds.), 'Spectral Classification and Multicolour Photometry', IAU Symp. 24, 6.

Jenkins, L. F.: 1952, General Catalogue of Trigonometric Stellar Parallaxes, Yale Univ. Obs., New Haven, Conn.

Jenkins, L. F.: 1963, Supplement to the General Catalogue of Trigonometric Stellar Parallaxes, Yale Univ. Obs., New Haven, Conn.

Kuiper, G. P.: 1942, Astrophys. J. 95, 201.

Wilson, R. E.: 1953, General Catalogue of Stellar Radial Velocities, Carnegie Inst. Washington, D. C., Publ. 601. 